\title{
Simulation-Based Evaluation of Variation in Left-Turn Paths in the Coordinated Intersection Management
}

\author{
Menglin Yang $\mathbb{D}^{1,2}$ Hao Yu $\mathbb{D}^{1,2}$ and Lu Bai $\mathbb{D}^{1,2}$ \\ ${ }^{1}$ School of Transportation, Southeast University, Nanjing 210096, China \\ ${ }^{2}$ Jiangsu Key Laboratory of Urban ITS, Nanjing 210096, China
}

Correspondence should be addressed to Hao Yu; seudarwin@gmail.com

Received 18 November 2021; Accepted 7 December 2021; Published 23 December 2021

Academic Editor: Wenxiang Li

Copyright (C) 2021 Menglin Yang et al. This is an open access article distributed under the Creative Commons Attribution License, which permits unrestricted use, distribution, and reproduction in any medium, provided the original work is properly cited.

Coordinated intersection management (CIM) has gained more attention with the advance of connected and autonomous vehicle technology. The optimization of passing schedules and conflict separation between conflicting vehicles are usually conducted based on the predefined travelling paths through the intersection area in the CIM. In real-world implementation, however, the diversity of turn paths exists due to multiple factors such as various vehicle sizes and automation control algorithms. The aim of this paper is to investigate how the variation in left-turn paths affects the feasibility and viability of optimal passing schedules, as well as the safety and efficiency of intersection operation. To do this, we start with identifying six typical left-turn paths to represent the variation. A scenario-based simulation is first conducted by using each of the paths as the nominal path. The optimal schedules and the corresponding alternative schedules are generated to calculate indicators for nominal performance, average performance, and robustness. The best path is selected in terms of schedule optimality and robustness. With schedules obtained by solving CIM models using the selected path, the left-turning CAVs are assumed to travel along one of the six paths randomly to simulate the path divergence. A surrogate safety measure, PET, is utilized to assess the safety of the intersection under CIM. The theoretical PET with the nominal path and the actual PET with the random path are calculated for each conflict event. Comparisons of two PET sets show the increase in conflict risk and vehicle delay. The conclusion can be drawn that the variation in left-turn paths causes the decline in safety level and travelling efficiency and should be considered in the CIM model to ensure safe and efficient implementation in the intersection.

\section{Introduction}

Connected and autonomous vehicles, which react faster than human drivers and have communication functions, provide a promising approach to improve traffic operations while maintaining the maximum safety level for urban traffic network. With the implementation of techniques including vehicle-to-vehicle (V2V)/vehicle-to-infrastructure (V2I) communication and vehicle automation, coordinated intersection management (CIM) or autonomous intersection management (AIM) is proposed as a new intersection management method for signal-free intersections under $100 \%$ connected and autonomous vehicle (CAV) environment [1], which indicates that vehicles communicate with intersections and/or other vehicles to cooperate to pass an intersection without collisions. CIM is commonly implemented in two approaches, including the centralized and decentralized way. In the centralized way, CIM achieves the intersection level objectives, such as ensuring collision avoidance, minimizing total delay, reducing air pollution by assigning the vehicles' entering sequences and reaching times at the intersection [2-10]. More specifically, an intersection manager (IM) is deployed to communicate with CAVs via infrastructure-to-vehicle (V2I) communication and coordinate their passing schedules through the intersection. Here, passing schedules include arrival times at the intersection and the travelling speed through the intersection of all approaching vehicles. In the decentralized way, CAVs usually have their own distributed controller for trajectory optimization and executing vehicle control. They 
communicate with ambient traffic or vehicles with conflicting movements to achieve consensus on collision-free trajectories [11-13]. The decentralized approach is also commonly applied in other scenarios such as cooperative metering $[14,15]$ and lane changing $[16,17]$. Obviously, the communication burden is lower than the centralized way, but the coordination might not be optimal in terms of intersection operation due to the deficiency of the global scope of all approaching vehicles. Therefore, in the study, we concentrate on the centralized approach of CIM.

As illustrated in Figure 1, the communication and coordination area of an intersection virtually consists of two functional zones: multiple buffer zones (BZs) where vehicles adjust their speeds to pass the intersection as scheduled by the IM and a conflict zone (CZ) where all conflict points exist. The CIM aims to resolve conflicts of different vehicle movements within the $\mathrm{CZ}$ by the effective allocation of the right of way to approaching vehicles and to achieve the operation objectives, for example, increasing throughput and reducing energy consumption simultaneously. Specifically, the IM arranges the passing sequences of conflicting vehicles, such as vehicles travelling straight with those making the left turn from the opposite direction, through their potential conflicting areas at the CZ. Here, passing sequences do not refer to the order by which conflicting vehicles enter the intersection, but the order by which vehicles arriving and travelling through the conflicting area overlapped by two paths. On-Board Units (OBUs) of CAVs receive the assigned arrival times at the $\mathrm{CZs}$ and desired travel speeds through the intersections and manipulate the vehicles to realize the IM's schedule.

Lane-based trajectories are usually applied in approaching lane setting, signal timing planning, and traffic conflict analysis at intersections [18-20]. Vehicle movement paths and conflict points within the $\mathrm{CZ}$ are also predefined in the formulation of the CIM problem according to the lane-based assumption in previous studies of CIM. For example, Levin and Rey [5] assumed that turning movements are circular lines with determined left-turn and rightturn radius and formulated the conflict-point separation algorithm at intersections based on the predefined movement paths. Li et al. [8] utilized the planes instead of lines to represent vehicle trajectories with the consideration of vehicle sizes, where turning movements were still presented as quarter-circles with determined radiuses. In the research of Yu et al. [7], an intersection area was separated by a grid of tiles where vehicle trajectories were determined and represented by different sets of tiles. The trajectories of turning vehicles were assumed to be elliptical arcs of predefined radiuses to identify the occupied tiles and occupying time durations. With the decentralized approach of CIM, Zhang and Cassandras [13] used the turning radius of the circular path linking the approaching and destination lane to calculate the desirable times for vehicles' making a turn, which was provided for coordinated control of vehicles passing through the intersection. With the lane-based assumption, CAVs only need to design and implement one-dimensional speed/acceleration profiles along the predefined paths according to the received schedules.

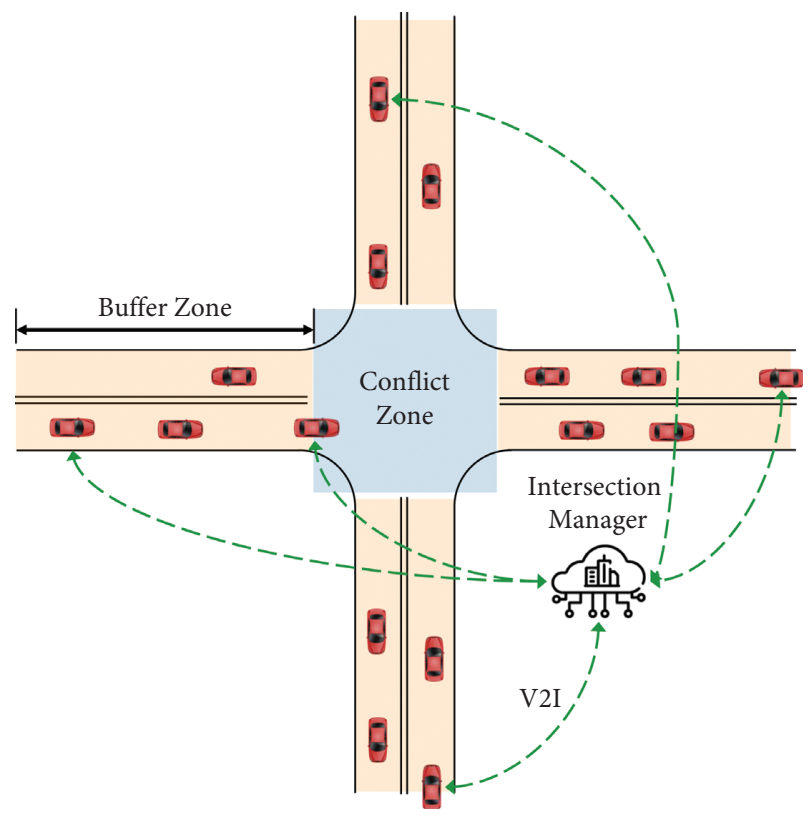

FIgURE 1: An exemplar scenario of CIM at a four-armed intersection.

However, the variation in vehicle turn paths is inevitable even for CAVs in real-world implementation. In the centralized CIM, in addition to target lane selection which is conducted by the IM, the controlling of CAVs through an intersection mainly consists of two tasks: trajectory planning and calculation and trajectory tracking and maneuvering. For straight travelling vehicles, one-dimensional speed/acceleration profiles are calculated and easy to track due to the few variations in the geometric path. For turning vehicles, however, the tasks are complicated since the geographical trajectory planning and calculation should generate both speed/acceleration and turning angle profiles, which needs to determine the turning pattern and the turning radius first. In this stage, the variety in vehicle sizes [21], planning algorithms [22], intersection geometric property [23-25], and desired turning speeds $[21,26,27]$ directly leads to different turning patterns and radiuses, referring to variation in both vehicle position and speed trajectories. In the trajectory tracking and maneuvering, the speed and turning angle are required to be adjusted simultaneously. Diversity in the control performance of different controllers and health status of motor drive systems also cause errors or failures in tracking planned turning trajectories in terms of geometric path and turning speed. Therefore, even with assigned passing schedules and target lanes, the actual turns may not be made along circular arcs linking approaching and target lanes or other predefined paths by the IM.

Due to the significance of predefined paths in conflicting relationship determination and subsequent conflict separation design and the inevitability of path divergence from the predefined ones, it is questioned whether the feasibility and viability of schedules and collision-free operation would remain. Therefore, it is of great importance to investigate two aspects: what effects the variation in turn paths has on the schedules and how the safety and travelling efficiency of 
the intersection could vary due to the path divergence. Since the left-turn path is longer and has more conflict points, this study concentrates on the variation in the left-turn path.

The centralized CIM scheduling problem is usually modelled as a mixed integer linear programming problem (MILP), with the passing sequences of conflicting vehicles as binary variables and arrival times at the intersection as continuous variables [5-7]. In the model formulation, the variations of turn paths are reflected in the model parameters. Hence, the primary trial is analysing the impact of the variations on the solutions (passing schedules) by the sensitivity analysis of the mathematical model. However, sensitivity analysis in the integer programming is still not mature due to the lack of optimality criteria, although it is well developed in the linear programming. Dawande and Hooker [28] developed a method of sensitivity analysis for MILP derived from the idea of inference duality to obtain the perturbations of parameters that reduce the optimal value by no more than a prespecified amount. Utilizing the inferencebased sensitivity analysis of MILP, Jia and Ierapetritou [29] proposed an integrated framework to address the issue of uncertainty in short-term scheduling problems of batch plants. However, the inference-based sensitivity analysis is not suitable for MILP problems in the CIM. The reason is that parameters in the corresponding MILP are derived and calculated from real-world information (i.e., geometric data of different turning paths). Those parameters have no direct physical meanings and are highly related to each other. Therefore, the achieved perturbations in those parameters from the inference-based sensitivity analysis are not useful for the real-world analysis such as feasibility of passing schedules. Besides, the inference-based sensitivity analysis addresses the MILP solved using the branch-and-bound methodology, making it difficult to be adopted due to computational complexity with the rise in the number of integer variables, which has a positive correlation with traffic demand through the intersection.

Without appropriate mathematical tools, it is impossible to investigate the variation issues in left-turn paths analytically. Therefore, we propose a scenario-based approach to evaluate the effects of variation in left-turn paths on travel efficiency and safety performance of CIM. Six typical left- turn paths are selected from two main turning patterns of different turning radius to be applied in the numerical tests. The scenario-based evaluation is conducted to assess the optimality and robustness of schedules obtained by using different left-turn paths as the nominal path. Based on the evaluation result, schedules are determined using the best path(s) and left-turning CAVs travel along the random leftturn paths according to the scheduled arrival times and desired speeds. Safety and travelling efficiency of the intersection are then estimated based on the schedules and the actual operation with path divergence, respectively, and compared to assess the effects of the variation in left-turn paths.

This paper is organized as follows: the scheduling model to optimize vehicle passing arrangements in the CIM is briefly described and analysed in Section 2 . Section 3 provides two simulation-based evaluation approaches for analysing the variation in left-turn paths. Section 4 presents the safety and efficiency analysis based on the CIM simulation results. Conclusive comments are listed, and future research directions are discussed in the last section.

\section{CIM Scheduling Model}

2.1. Original Model. In this paper, the mathematical model used for vehicle passing schedule optimization in the CIM is formulated based on the vehicle movement paths within the intersection [5]. The paths (denoted by $\rho$ ) are discretized by critical points, and the critical points contain two parts: the starting points (denoted by $o$ ) and ending points (denoted by $f$ ) of the allowed paths on the boundary of the conflict zone and the conflict points (denoted by $c$ ) where conflicting paths overlap. The exemplar intersection is illustrated in Figure 2, along with all movements, paths, and critical points within the CZ.

The objective to minimize the integrated passing time of the intersection over all vehicles in one optimization horizon, and other constraints in the MILP model are as follows:

$$
z=\min \sum_{i \in \Omega} p_{i} t_{i}(f),
$$

subject to

$$
\begin{gathered}
t_{i}(c)+\tau_{i}(c)-t_{j}(c) \leq\left(1-\delta_{\mathrm{ij}}\right) M, \forall i, j \in \Omega, c=\rho_{i} \cap \rho_{j}, \rho_{i} \neq \rho_{j}, \\
t_{j}(c)+\tau_{j}(c)-t_{i}(c) \leq \delta_{\mathrm{ij}} M, \forall i, j \in \Omega, c=\rho_{i} \cap \rho_{j}, \rho_{i} \neq \rho_{j}, \\
t_{i}(o)+\tau_{i}(o) \leq t_{j}(o), \forall i, j \in \Omega, o \in \rho_{i} \rho_{i}=\rho_{j}, \\
t_{i}(f)+\tau_{i}(f) \leq t_{j}(f), \forall i, j \in \Omega, f \in \rho_{i}, \rho_{i}=\rho_{j}, \\
\frac{d_{i}(o, f)}{\bar{U}_{i}} \leq t_{i}(f)-t_{i}(o) \leq \frac{d_{i}(o, f)}{\underline{U}_{i}}, \forall i \in \Omega, o, f \in \rho_{i},
\end{gathered}
$$




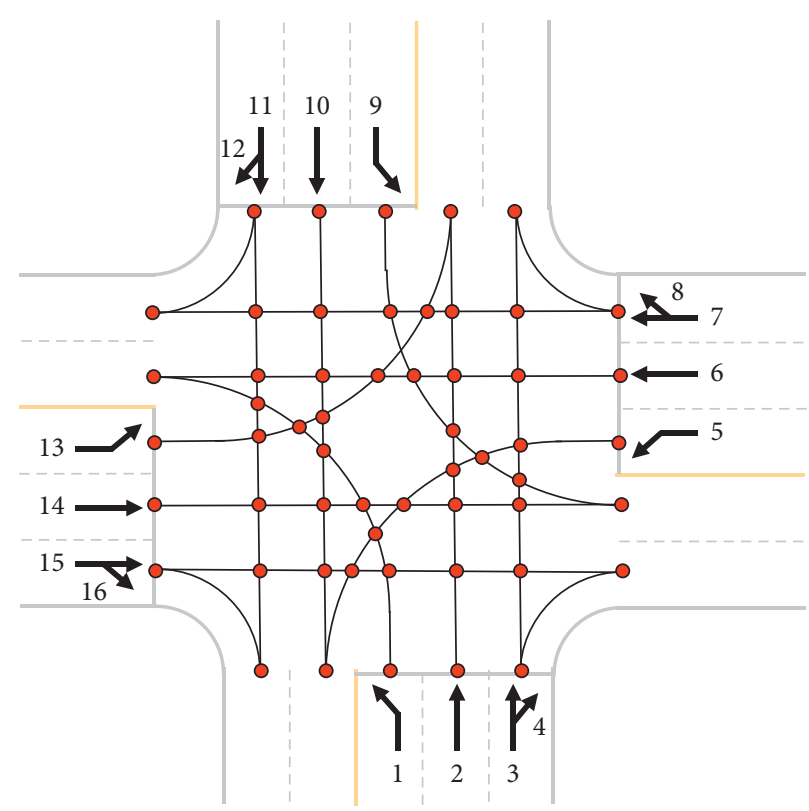

- Vehicle paths $\rho$

$\longleftarrow$ Vehicle movements

- Critical points $o, f$ or $c$

FIGURE 2: A four-armed exemplar intersection.

$$
\begin{aligned}
& \frac{t_{i}(f)-t_{i}(o)}{d_{i}(o, f)}=\frac{t_{i}(c)-t_{i}(o)}{d_{i}(o, c)}, \forall i \in \Omega, o, f \in \rho_{i}, c=\rho_{i} \cap \rho_{j}, \rho_{i} \neq \rho_{j}, \\
& t_{i}(o) \geq \max \left\{t_{i}(B)+e_{i}, h_{i}\right\}, \forall i \in \Omega, o \in \rho_{i}, \\
& t_{i}(\cdot) \geq 0, \forall i \in \Omega, \\
& \delta_{\mathrm{ij}}=\{0,1\}, \forall i, j \in \Omega, \rho_{i} \neq \rho_{j},
\end{aligned}
$$

where $\Omega$ is the vehicle set consisting of all vehicles in one optimization horizon and $i$ and $j$ are the indices of vehicles. $\rho$ represents any path illustrated in Figure 2. $p_{i}$ represents the priority of vehicle passing sequences. $t_{i}(\bullet)$ refers to the arrival time at one critical point of vehicle $i$ and $\bullet$ can be either a starting point $(o)$, an ending point $(f)$, or a conflict point $(c)$ of one path. $\tau_{i}(\bullet)$ refers to the reservation time duration of such a point for vehicle $i$, consisting of the encroachment time of the point by vehicle $i$ and the predefined clearance time, as expressed by equation $(11) . d_{i}(o, \bullet)$ is the distance from the starting point to another critical point along path $\rho_{i} . t_{i}(B)+e_{i}$ denotes the earliest possible arrival time at the $\mathrm{CZ}$ of vehicle $i$, where $t_{i}(B)$ is its arrival time at the buffer zone and $e_{i}$ is the estimated shortest time travelling through the BZ. $\delta_{i j}$ is a binary variable indicating the passing sequence through the conflict point of vehicles $i$ and $j$.

$$
\tau_{i}=\frac{L_{i}}{u_{i}}+g=\frac{L_{i}\left(t_{i}(c)-t_{i}(o)\right)}{d_{i}(o, c)}+g, \forall i \in \Omega, o \in \rho_{i}, c=\rho_{i} \cap \rho_{j}, \gamma_{i} \neq \gamma_{j},
$$

where $L_{i}$ denotes the distance that vehicle $i$ entirely passes through a point (i.e., $c=\rho_{i} \cap \rho_{j}$ ) along path $\rho_{i} . g$ represents the minimum clearance time. The detailed calculation process of $L_{i}$ can be found in [5].

In general, equations (2) and (3) represent the determination of passing sequences at conflict point $c$. Specifically, when $\delta_{i j}=1$, equation (2) works, while equation (3) is inactive, indicating that vehicle $i$ passes ahead of vehicle $j$ over point $c$. When $\delta_{i j}=0$, equation (3) works, while equation (2) is inactive, indicating the reverse passing sequence. Equations (4) and (5) constrain the car-following behaviors of vehicles on the same path. The upper and lower speed boundaries of the intersection are expressed by equation (6), and constant speed limitation within the conflict zone is indicated by equation (7). Equation (8) indicates the estimated earliest arrival time at the conflict 
zone of vehicle $i$. Basic constraints of variables are expressed by equations (9) and (10).

2.2. Reformulation. To clearly display the relationship between MILP parameters and factors at the real-world level, we reformulate the original MILP (equations (1)-(10)). With no priority of any vehicle, let us set $p_{i}=1, \forall i \in \Omega$. We take $x_{i}=t_{i}(f)-t_{i}(o)$ and $t_{i}$ (short for $t_{i}(o)$ ) as the continuous variables. $t_{i}(c)$ is transformed to be the linear combination of $x_{i}$ and $t_{i}$ based on equation (7). Therefore, the problem is reformulated as

$$
z=\min \sum_{i \in \Omega}\left(t_{i}+x_{i}\right)
$$

subject to

$$
\begin{aligned}
-t_{i}+t_{j}-\left(\alpha_{\mathrm{ic}}+\beta_{i}\right) x_{i}+\alpha_{\mathrm{jc}} x_{j}-M \delta_{\mathrm{ij}} & \geq g-M, \forall i, j \in \Omega, c=\rho_{i} \cap \rho_{j}, \rho_{i} \neq \rho_{j}, \\
t_{i}-t_{j}+\alpha_{\mathrm{ic}} x_{i}-\left(\alpha_{\mathrm{jc}}+\beta_{j}\right) x_{j}+M \delta_{\mathrm{ij}} & \geq g, \forall i, j \in \Omega, c=\rho_{i} \cap \rho_{j}, \rho_{i} \neq \rho_{j}, \\
-\left[1+\frac{L_{i}}{L}\right] \cdot t_{i}+t_{j} & \geq-\frac{L_{i}}{L} \cdot t_{j}(B)+g, \forall i, j \in \Omega, \rho_{i}=\rho_{j}, \\
-t_{i}+t_{j}-\left[1+\beta_{i}\right] \cdot x_{i}+x_{j} & \geq g, \forall i, j \in \Omega, \rho_{i}=\rho_{j}, \\
x_{i} & \geq \underline{\tau_{i}}, \forall i=1, \ldots, 7, \\
-x_{i} & \geq-\bar{\tau}_{i}, \forall i=1, \ldots, 7, \\
t_{i} & \geq \gamma_{i}, \forall i=1, \ldots, 7, \\
\delta_{\mathrm{ij}} & =\{0,1\}, \forall i, j \in \Omega, c=\rho_{i} \cap \rho_{j}, \rho_{i} \neq \rho_{j},
\end{aligned}
$$

where $L$ is the length of the buffer zone, $\alpha_{\mathrm{ic}}=d_{i}(o, c) / d_{i}(o, f), \quad \beta_{i}=L_{i} / d_{i}(o, f), \quad \tau_{i}=d_{i}(o, f) / \bar{U}_{i}$, $\bar{\tau}_{i}=d_{i}(o, f) / \underline{U}_{i}$, and $\gamma_{i}=\max \left\{t_{i}(B)+e_{i}, h_{i}\right\}$.

Equations (16) and (17), which are derived from equation (6), represent the upper and lower bounds of $x_{i}$. Vehicle passing arrangements through the $\mathrm{CZ}$ can be interpreted from the variable values obtained by solving the MILP problem. To be specific, the passing sequences are indicated directly by values of $\delta_{i j}$, the arranged arrival times at the CZ are achieved directly by values of $t_{i}$, and vehicle cruise speeds $\left(v_{i}\right)$ through the $\mathrm{CZ}$ can be calculated as follows:

$$
v_{i}=\frac{d_{i}(o, f)}{x_{i}}, \forall i \in \Omega .
$$

2.3. Variation in Left-Turn Paths. Turning pattern and radius are two main features of a turn path. Left-turn patterns are classified into two types: two-stage pattern (straight and turning) and one-stage pattern (directly turning) to generally model the formation of path diversity [30]. Here, six typical left-turn paths are selected to represent the variation of left-turn paths, including three of the two-stage pattern (Paths C1, C2, and C3 in Figure 3(a)) and three of the onestage pattern (Paths E1, E2, and E3 in Figure 3(b)), where $w$ and $W$ refer to the width of a vehicle and a lane, respectively. The middle paths (Paths C2 and E2) are on the centerline of lanes, while the external paths (Paths $C 1$ and E1) and the internal paths (Paths $C 3$ and E3) are the paths where vehicles travel along the boundaries of the lane. For each pattern, the most common condition (travelling on the centerline) and two extreme conditions (travelling laterally along the two sides of the lane) are presented and considered in the study. The locations of related conflict points also vary with the deviation of paths. These variations are mainly reflected by the changes of $d_{i}(o, \bullet)$ and accordingly the changes in values of parameters $\alpha_{i c}, \beta_{i}$, and $\tau_{i}$ in the MILP.

\section{Simulation-Based Evaluation}

3.1. A Scenario-Based Approach. With the selected left-turn paths, we propose a scenario-based approach to analyse the efficiency and robustness of schedules solved from the CIM model, to investigate whether a schedule obtained with a predefined path can accommodate the variations in actual paths and still holds valid. The proposed scenariobased approach (see Figure 4) comprises the following steps:

Step 1. Six scenarios $k(k=1,2, \ldots, 6)$ are defined where the typical left-turn paths (Paths $C 1, C 2, C 3, E 1, E 2$, and E3) are used as the nominal path, respectively, and the corresponding parameter sets $\left(\alpha_{i c}, \beta_{i}\right.$, and $\left.\tau_{i}\right)$ of the MILP problem are calculated with the geometry of different left-turn paths under each scenario.

Step 2. An MILP problem is solved based on the nominal values of the varying parameter set $\left(\alpha_{i c}, \beta_{i}\right.$, and $\tau_{i}$ ) from the scenario $k$. A set of optimal fixed sequences of vehicle passing through the $\mathrm{CZ}\left(\delta_{i j}\right)$ is obtained, and the solution is denoted as Schedule $k$. The objective 


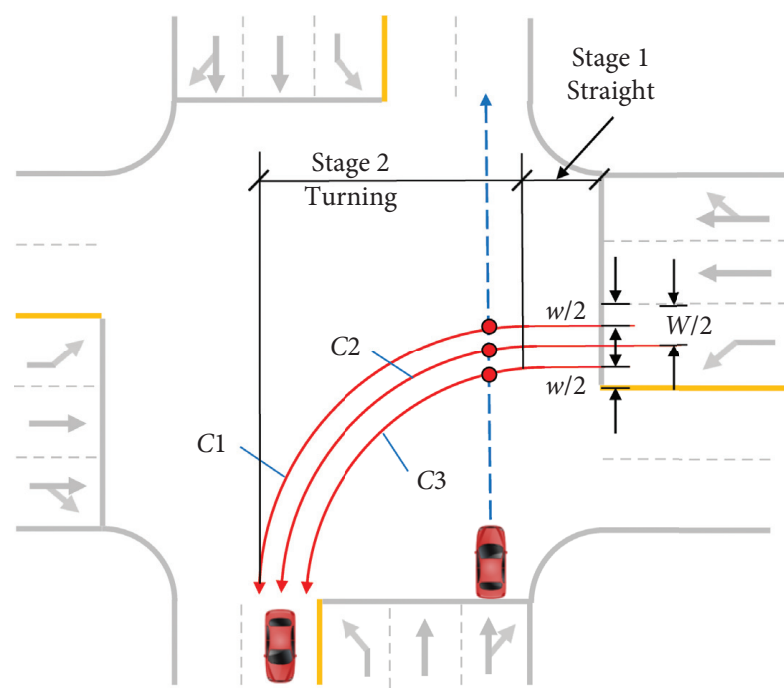

(a)

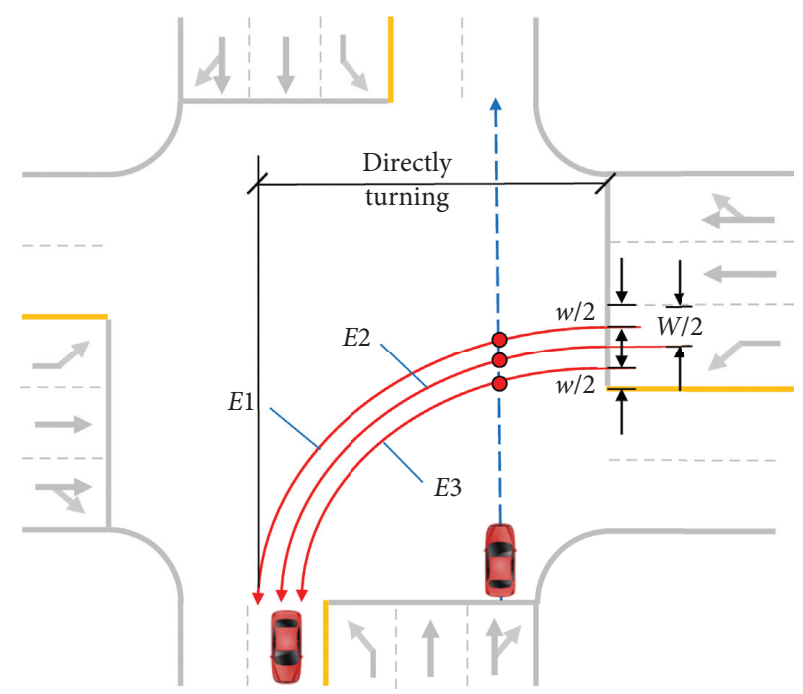

(b)

Figure 3: Typical left-turn paths. (a) Two-stage left-turn paths. (b) One-stage left-turn paths.

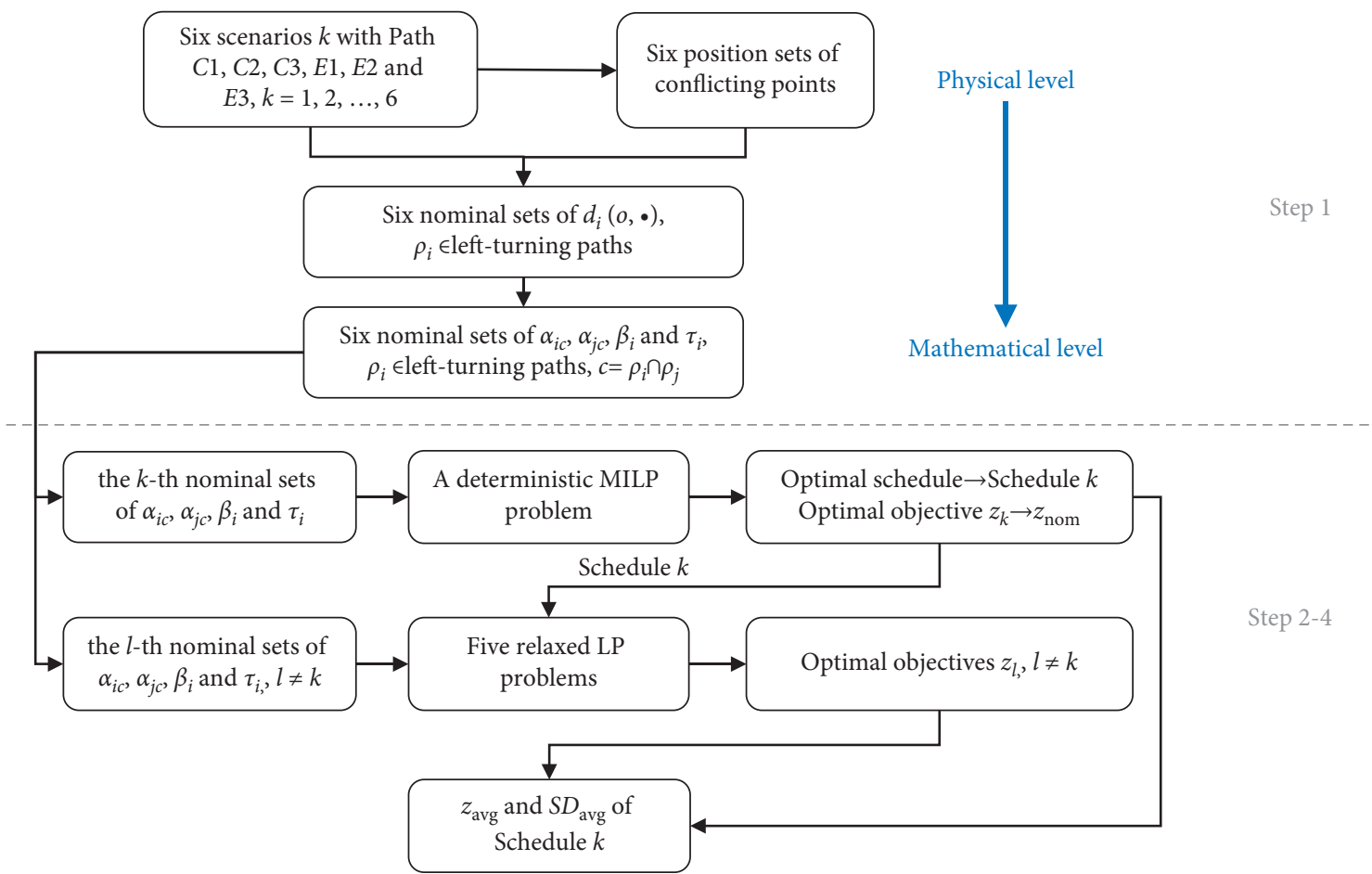

Figure 4: Procedure of the scenario-based approach.

value $\left(z_{k}\right)$ is recorded as the nominal performance $\left(z_{\text {nom }}\right)$ of this schedule.

Step 3. Using the optimal sequences of Schedule $k$ in Step 2, the MILP problem is relaxed to be LP problems using parameter sets of alternative paths from all other scenarios $l$ $(l \neq k)$. The alternative schedules $l$ and the optimized objective values $\left(z_{l}\right)$ are obtained by solving those LP problems.
Step 4 . The average performance and robustness metric of Schedule $k$ are calculated. To be specific, the average performance of Schedule $k\left(z_{\text {avg }}\right)$ is calculated as follows:

$$
z_{\text {avg }}=\frac{z_{k}+\sum_{l} z_{l}}{p_{\text {tot }}}
$$


where $p_{t o t}$ is the total number of scenarios. The average performance $\left(z_{\text {avg }}\right)$ is used to access the performance of a schedule over the entire variation interval.

The standard deviation (SD) is applied as the robustness metric for Schedule $k$. Like Jia and Ierapetritou [29], the SD is defined as

$$
\mathrm{SD}_{\mathrm{avg}}=\sqrt{\sum_{k} \frac{\left(z_{k}-z_{\mathrm{avg}}\right)^{2}}{\left(p_{\mathrm{tot}}-1\right)}} .
$$

As expressed by equation (12), the objective $z$ is the integration of all vehicles' arranged arrival time $\left(t_{i}\right)$ at the intersection and travelling time $\left(x_{i}\right)$ through the $\mathrm{CZ}$ in one optimization horizon. Lower $z_{\text {nom }}$ represents the earlier, and lower $z_{\text {avg }}$ represents the earlier average integrated passing time of vehicles over all scenarios with different left-turn paths, indicating the higher travelling efficiency of the intersection. Lower $S D_{\text {avg }}$ of a schedule represents less fluctuation in the objective value under different left-turn paths. With the same vehicle passing sequences, this presents how close the passing arrangements $\left(t_{i}\right.$ and $\left.x_{i}\right)$ under different scenarios are to each other, which indicates the robustness of a schedule in terms of travelling efficiency. To evaluate the effects of the variation in left-turn paths on CIM, simulations are conducted under different traffic demand levels by the scenario-based approach using the six typical paths mentioned above. To eliminate the impacts of vehicle arrival times and vehicle numbers of different cases on the values of schedule performance metrics, we utilize travel delay per vehicle as the objective function of the MILP, which is modified as

$$
z^{\prime}=\frac{1}{s} \min \sum_{i \in \Omega}\left[t_{i}+x_{i}-\left(t_{i}(B)+e_{i}+\frac{d_{i}(o, f)}{\bar{U}_{i}}\right)\right], \forall i \in \Omega,
$$

where $s$ is the total number of vehicles in $\Omega$.

Nominal performance $\left(z_{\text {nom }}^{\prime}\right)$, average performance $\left(z_{\text {avg }}^{\prime}\right)$, and robustness metric ( $\left.S D_{\text {avg }}^{\prime}\right)$ of six schedules are calculated to evaluate whether a schedule obtained by using a certain path outperforms the others. This selected path will be used for further evaluation.

Note that higher $S D_{\text {avg }}$ values can indicate that the arranged arrival times vary seriously of at least some vehicles. The lower values, however, cannot refer to the less variation in the arranged arrival times of each vehicle. Therefore, the PET-based approach is proposed and then proposed to analyse the effects of path variation on individual vehicles and conflicts.

3.2. A PET-Based Approach. As demonstrated in equations (13) and (14), the potential conflict between two conflicting vehicles is resolved by determining the passing sequence and minimum clearance time of the conflict point based on the predefined paths. The path divergence between theoretical ones in the CIM models and actual ones in the schedule implementation would affect the actual locations and clearance time of the conflict points, raising safety concerns of failures in conflict separation, leading to collisions subsequently. To evaluate the traffic collision risks considering the variation in left-turn paths, Postencroachment Time (PET) is utilized in this study. Peesapati, Hunter, and Rodgers [31, 32] evaluated the effectiveness of PET as an indicator of safety assessment and as a surrogate measure for the propensity of crashes between left-turning vehicles and opposing through vehicles at four-armed signalized intersections.

As illustrated in Figure 5, PET is the difference between the time when the first vehicle (veh $i$ ) ends encroachment over the conflict point and the second vehicle (veh $j$ ) arrives the point, representing the proximity of conflicting vehicles to their actual crossing point and it has no requirement on the collision course $[33,34]$. Only two timestamps are required to compute PET, and it has a definite boundary to differentiate a crash from a noncrash event. A PET value of 0 implies the occurrence of a collision, and the closer the value of PET is to 0 , the higher the conflict risk is. In this study, two threshold values of PET play an important role in establishing its correlation with collisions as well as traffic safety. In the CIM model, a minimum clearance time $g$ is set to ensure collision-free operation. Therefore, when the PET between a pair of conflicting vehicles is lower than $g$, it is considered as a serious conflict. The other threshold is set to filter out the nonconflict events from the analysis to eliminate the bias in the safety evaluation results [35], which usually takes 3 seconds [36].

Further simulations are conducted under different traffic demand levels where the schedules are obtained by using the path selected by the scenario-based approach, and leftturning vehicles are assumed to travel along one of the six typical paths randomly. All potential conflict events involving left-turning vehicles are collected, and two PETs are captured for each event, including theoretical PET calculated based on the nominal path used in the model, denoted by $\mathrm{PET}_{t}$, and actual PET calculated based on the actual travel path, denoted by $\mathrm{PET}_{a}$. Here, a potential conflict event refers to the event when two vehicles travelling on conflicting movements occupy the CZ simultaneously. Two sets of PETs will be compared and analysed to evaluate the effects of the variation in left-turn paths on intersection safety and travel efficiency.

\section{Numerical Simulation and Result Analysis}

Simulations are conducted in MATLAB R2021a, including random vehicle arrival generation and CIM operation. The MILP model and the relaxed LP problems are formulated and organized as the standard form in MATLAB, solved by the build-in Mixed Integer Programming (MIP) solver in GAMS [37].

In the simulations, the exemplar intersection is deadlocked when the traffic demand exceeds $900 \mathrm{veh} / \mathrm{h} / \mathrm{lane}$, and the vehicle arrives sparsely when the traffic demand falls below $500 \mathrm{veh} / \mathrm{h} / \mathrm{lane}$, in which the effects of variations in the left-turn path can hardly be identified clearly. As a result, the traffic volume on straight movements is set from 500 to 


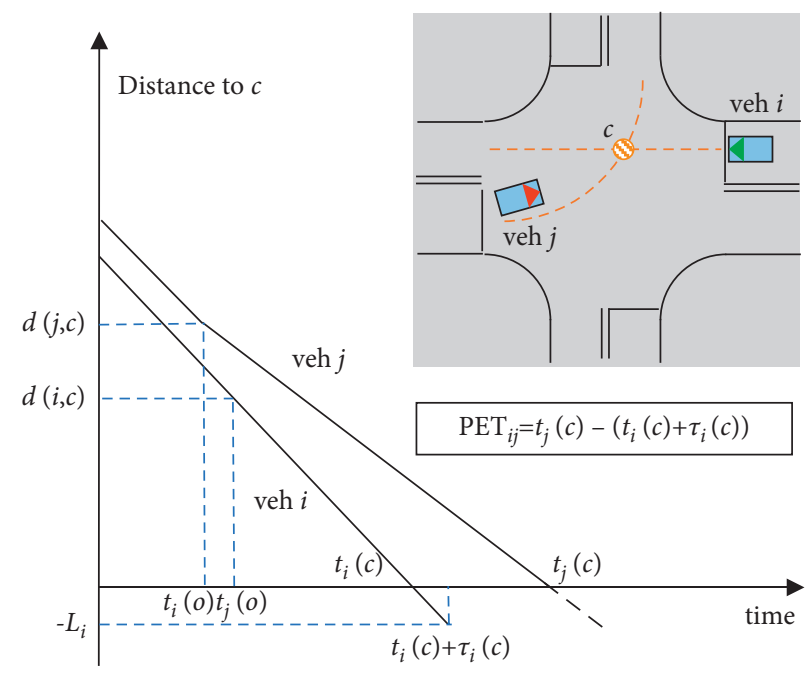

Figure 5: Traffic conflict diagram for PET.

$900 \mathrm{veh} / \mathrm{h} /$ lane with a $100 \mathrm{veh} / \mathrm{h} /$ lane step. Left-turn and right-turn volumes are both set to be $20 \%$ of the straight traffic volume. For each condition, the simulation runs for 150 minutes. The minimum clearance time $g$ is set to be 0.8 seconds, and the threshold to distinguish traffic conflict and nonconflict events is 3 seconds.

4.1. Travelling Efficiency and Robustness with Different LeftTurn Paths. The CIM process is conducted in a rollingforward approach, and the indicators including nominal performance $\left(z_{\text {nom }}^{\prime}\right)$, average performance $\left(z_{\text {avg }}^{\prime}\right)$, and robustness metric $\left(S D_{\text {avg }}^{\prime}\right)$ are collected for each optimization horizon. Table 1 demonstrates the average values of nominal and average performance ( $z_{\text {nom }}^{\prime}$ and $z_{\text {avg }}^{\prime}$ ) of all cases under each traffic demand level, where "Sched." is short for "Schedule." The $S D_{\text {avg }}$ of six schedules under all traffic demand levels are illustrated in Figure 6.

As listed in Table 1, Schedule 3 and Schedule 5 generally have both higher nominal performances and average performances (lower average delay per vehicle) than others across all traffic demand levels, indicating higher travelling efficiency of vehicle passing schedules. Considering the $S D_{\text {avg }}^{\prime}$ illustrated in Figure 6 , Schedule 5 provides better solutions than Schedule 3 in terms of robustness metric, indicating better accommodation to the variation in leftturn paths. Therefore, Path E2, as the nominal path, and the corresponding parameters will be used when modelling the CIM and formulating the MILP problem in the following simulation.

Schedule 1 and Schedule 4 have obvious worse robustness than others, as shown in Figure 5. The corresponding left-turn paths are Path $C 1$ and Path E1, which have the largest turning radiuses among each turning pattern. The optimal schedules obtained with longer nominal paths reserve more time for left-turning vehicles passing through conflict points as well as the intersection, of which the passing sequences are more likely not to be optimal for scenarios with shorter nominal paths, leading to more fluctuation in the average delay among different schedules. On the other side, although the optimal schedules obtained with shorter nominal paths reserve less time for left-turning vehicles, the passing sequences may be still optimal for scenarios with longer nominal paths since the clearance time can be reduced as long as it satisfies the minimum clearance time.

4.2. Safety and Travelling Efficiency with the Path Divergence. In this section, $\mathrm{PET}_{t}$ is calculated from schedules obtained by using Path E2 as the nominal path. Figure 7 displays the distribution of potential conflict events in terms of their $\mathrm{PET}_{a}$ values against the increases compared to their $\mathrm{PET}_{t}$. Some serious conflicts are observed, of which $\mathrm{PET}_{a}$ is lower than the minimum clearance time. To eliminate the effects of suboptimality of solutions from the solver, conflicts of which $\mathrm{PET}_{a}<0.8$ and those of which $\mathrm{PET}_{t}<0.8$ are extracted and compared in Table 2. Conflicts of which $\mathrm{PET}_{t}<0.8$ are caused by the solver and its solving algorithms. The obvious increases in both the numbers of conflicts with $\mathrm{PET}_{a}<0.8$ and their percentages across all traffic demand levels demonstrate higher collision risk at the intersection when the variation in the left-turn path exists but is not considered in the scheduling.

Another interesting change is the increase in the nonconflict events (PET >3) with rising traffic demand, as shown in Table 2. With the increased traffic volume, more potential conflicts involving the same vehicle need to be resolved simultaneously in one optimization horizon, and the solution might lead to higher PET values of some potential conflict events to ensure conflict separation for all vehicles.

After filtering out the nonconflict events, the distributions of conflicts under different traffic demand levels are demonstrated in Figure 8 to depict the fluctuations in conflict risk with the actual path divergence from the nominal path. The conflict numbers of which $\mathrm{PET}_{a}$ decreases compared to $\mathrm{PET}_{t}$ clearly surpass those of which $\mathrm{PET}_{a}$ increases under each traffic demand level. The overall effect of 
TABLE 1: Travelling efficiency performance of different nominal left-turn paths.

\begin{tabular}{|c|c|c|c|c|c|c|}
\hline $\begin{array}{c}\text { Traffic } \\
\text { demand } \\
\text { (veh/h/ } \\
\text { lane) }\end{array}$ & Sched. 1 (Path C1) & Sched. 2 (Path C2) & Sched. 3 (Path C3) & Sched. 4 (Path E1) & Sched. 5 (Path E2) & Sched. 6 (Path E3) \\
\hline 500 & 0.488 & 0.487 & 0.481 & 0.487 & 0.485 & 0.484 \\
\hline $500 z_{\text {avg }}^{\prime}$ & 0.503 & 0.490 & 0.483 & 0.491 & 0.482 & 0.483 \\
\hline $600 z_{\text {nom }}^{\prime}$ & 0.653 & 0.652 & 0.651 & 0.653 & 0.652 & 0.658 \\
\hline $600 \quad z_{\text {avg }}^{\prime}$ & 0.682 & 0.661 & 0.653 & 0.667 & 0.655 & 0.653 \\
\hline 700 & 0.871 & 0.877 & 0.876 & 0.874 & 0.878 & 0.879 \\
\hline $700 z_{\text {avg }}^{\prime}$ & 0.908 & 0.890 & 0.878 & 0.894 & 0.880 & 0.876 \\
\hline $800 z_{\text {nom }}^{\prime o}$ & 1.090 & 1.096 & 1.092 & 1.093 & 1.093 & 1.103 \\
\hline $800 \quad z_{\text {avg }}^{\prime}$ & 1.129 & 1.109 & 1.096 & 1.113 & 1.098 & 1.098 \\
\hline $900 z_{\text {nom }}^{\prime}$ & 1.924 & 1.928 & 1.927 & 1.927 & 1.929 & 1.941 \\
\hline $900 z_{\text {avg }}^{\prime}$ & 1.977 & 1.947 & 1.935 & 1.952 & 1.936 & 1.937 \\
\hline
\end{tabular}

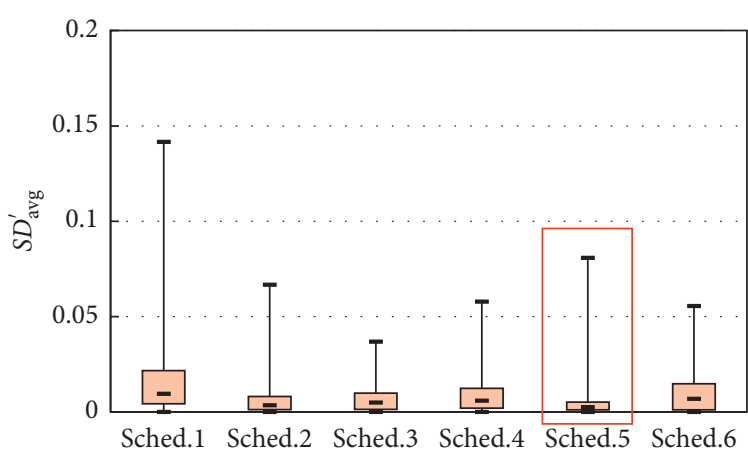

(a)

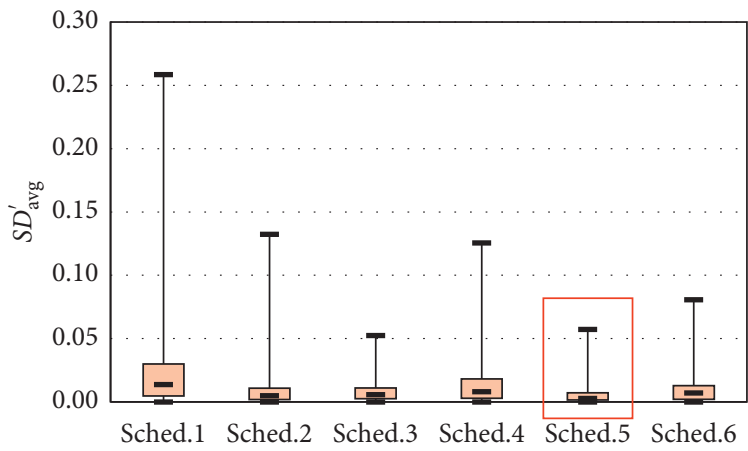

(c)

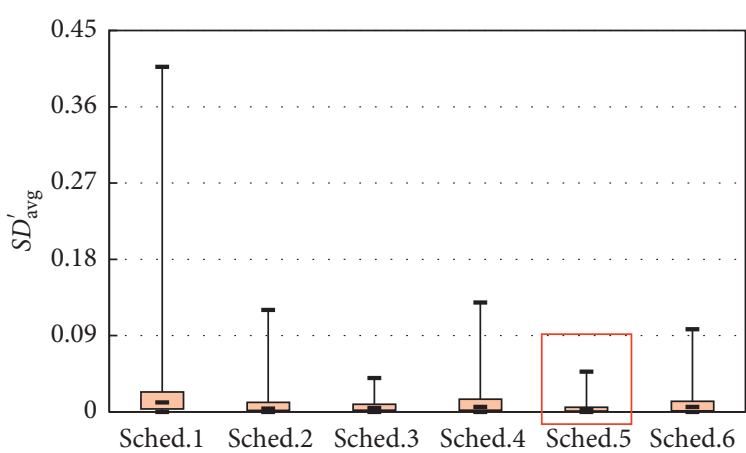

(b)

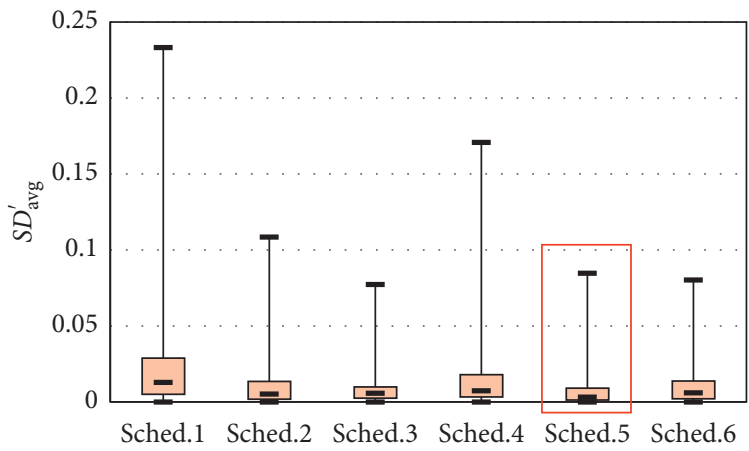

(d)

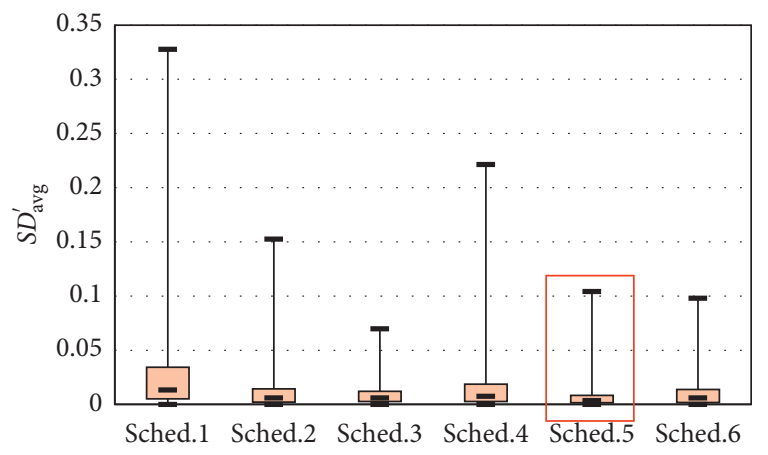

(e)

FiguRE 6: Schedule robustness of different nominal left-turn paths. (a) $500 \mathrm{veh} / \mathrm{h} / \mathrm{lane}$. (b) $600 \mathrm{veh} / \mathrm{h} / \mathrm{lane}$. (c) $700 \mathrm{veh} / \mathrm{h} / \mathrm{lane}$. (d) $800 \mathrm{veh} / \mathrm{h} /$ lane. (e) $900 \mathrm{veh} / \mathrm{h} / \mathrm{lane}$. 


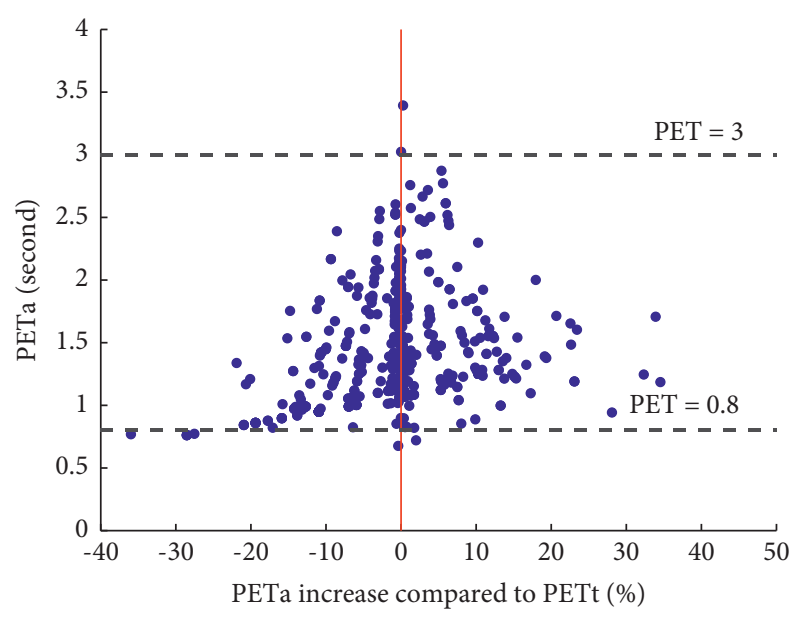

(a)

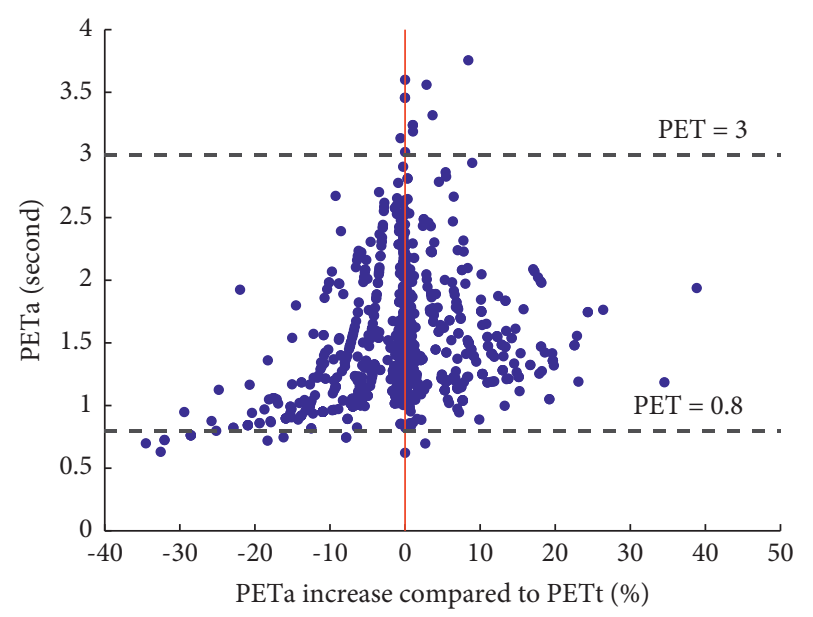

(c)

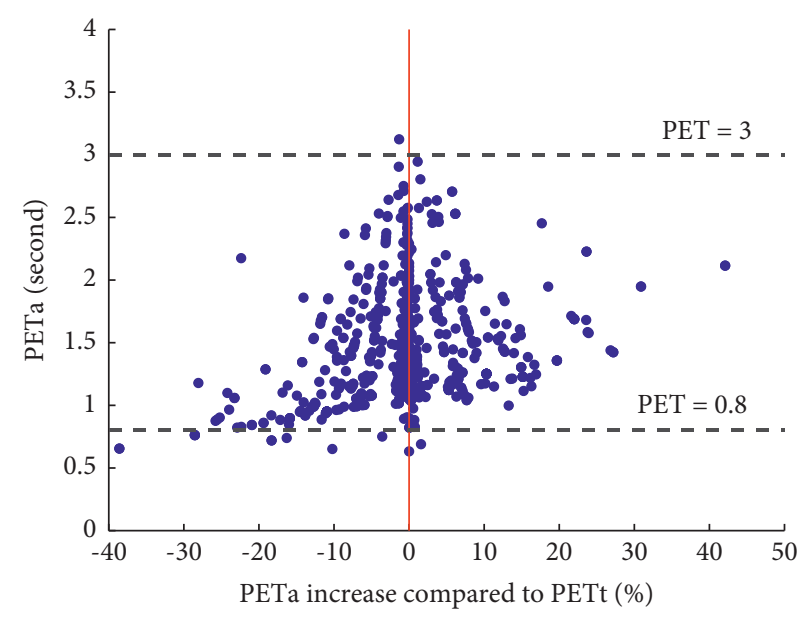

(b)

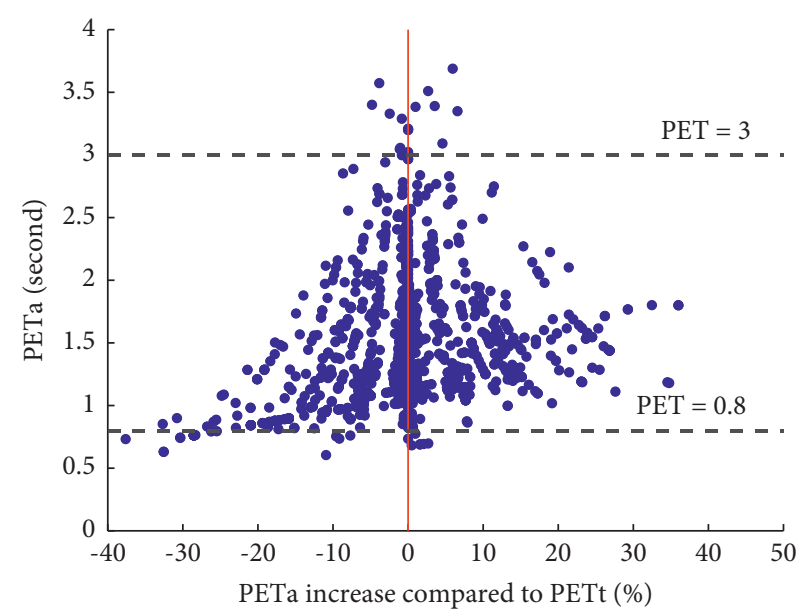

(d)

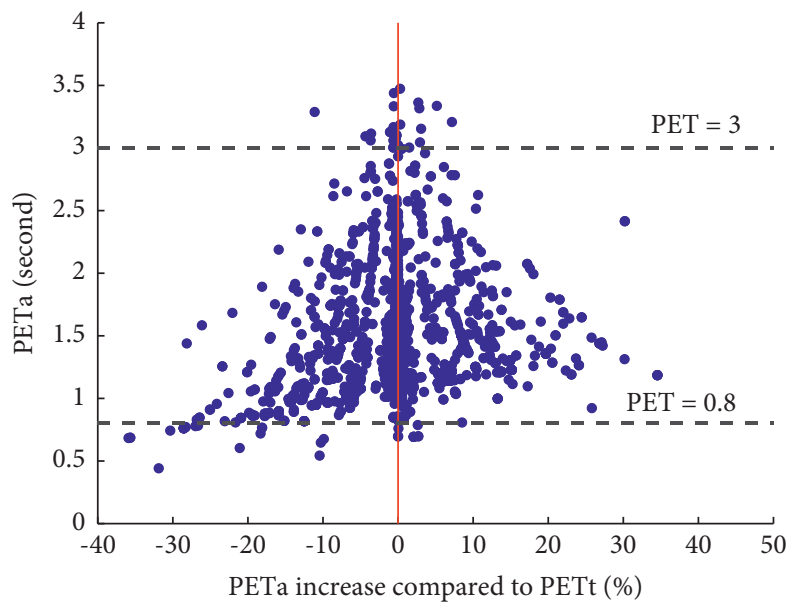

(e)

FIGURE 7: Relationships between $\mathrm{PET}_{a}$ and $\mathrm{PET}_{a}$ increase compared to $\mathrm{PET}_{t}$. (a) $500 \mathrm{veh} / \mathrm{h} / \mathrm{lane}$. (b) 600 veh/h/lane. (c) 700 veh/h/lane. (d) $800 \mathrm{veh} / \mathrm{h} / \mathrm{lane}$. (e) $900 \mathrm{veh} / \mathrm{h} / \mathrm{lane}$.

actual path divergence on safety is negative since the decrease of $\mathrm{PET}_{a}$ indicates the increase in conflict/collision risk. Hence, the variations in the left-turn path should be seriously considered in the scheduling to improve safety.
Apart from the safety issue, the travelling efficiency is also jeopardized due to the path divergence. During CAVs travelling across the intersection according to the schedules, OBUs are also monitoring the movements of surrounding 
TABLE 2: Conflicts of which $\mathrm{PET}_{t}$ and $\mathrm{PET}_{a}$ are beyond two thresholds.

\begin{tabular}{|c|c|c|c|c|c|c|}
\hline \multicolumn{2}{|c|}{ Thresholds } & \multirow{2}{*}{$\begin{array}{c}500 \mathrm{veh} / \mathrm{h} / \text { lane } \\
4\end{array}$} & \multirow{2}{*}{$\frac{600 \mathrm{veh} / \mathrm{h} / \text { lane }}{4}$} & \multirow{2}{*}{$\begin{array}{c}700 \mathrm{veh} / \mathrm{h} / \text { lane } \\
2\end{array}$} & \multirow{2}{*}{$\begin{array}{c}800 \mathrm{veh} / \mathrm{h} / \text { lane } \\
8\end{array}$} & \multirow{2}{*}{$\begin{array}{c}900 \mathrm{veh} / \mathrm{h} / \text { lane } \\
17\end{array}$} \\
\hline & Number & & & & & \\
\hline $\mathrm{PEI}_{t}<0.8 \mathrm{sec}$ & Percentage & 0.74 & 0.53 & 0.19 & 0.56 & 1.00 \\
\hline \multirow{2}{*}{$\mathrm{PET}_{a}<0.8 \mathrm{sec}$} & Number & 9 & 10 & 13 & 27 & 29 \\
\hline & Percentage & 1.65 & 1.34 & 1.23 & 1.92 & 1.71 \\
\hline \multirow{2}{*}{$\mathrm{PET}_{t}>3 \mathrm{sec}$} & Number & 2 & 2 & 11 & 19 & 23 \\
\hline & Percentage & 0.37 & 0.27 & 1.04 & 1.34 & 1.35 \\
\hline \multirow{2}{*}{$\mathrm{PET}_{a}>3 \mathrm{sec}$} & Number & 2 & 2 & 11 & 16 & 26 \\
\hline & Percentage & 0.37 & 0.27 & 1.04 & 1.14 & 1.54 \\
\hline
\end{tabular}

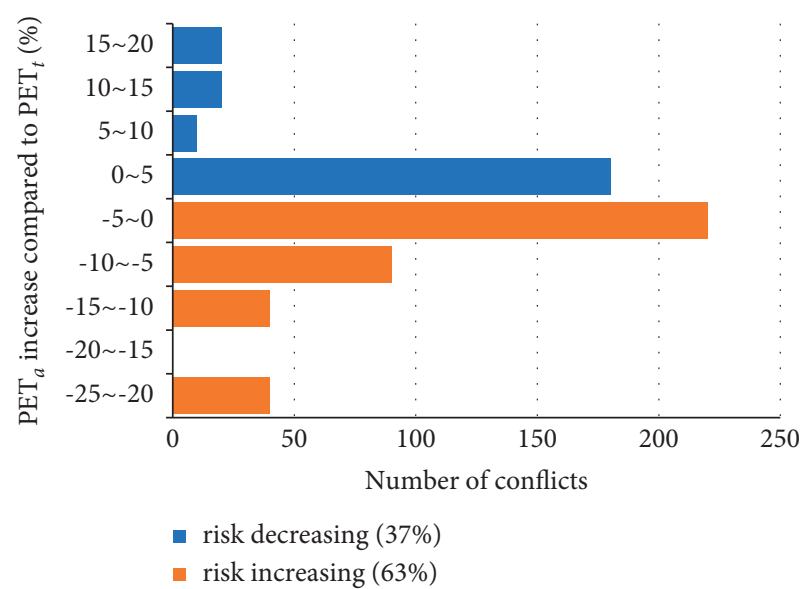

(a)

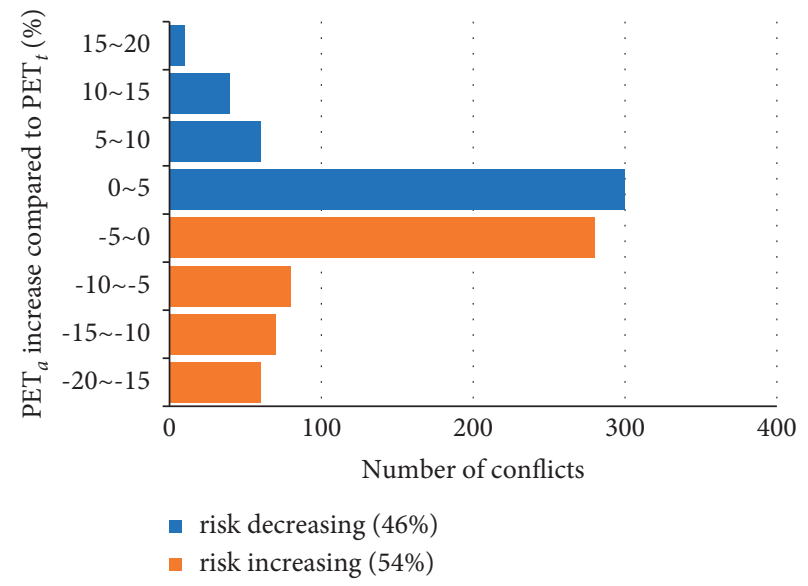

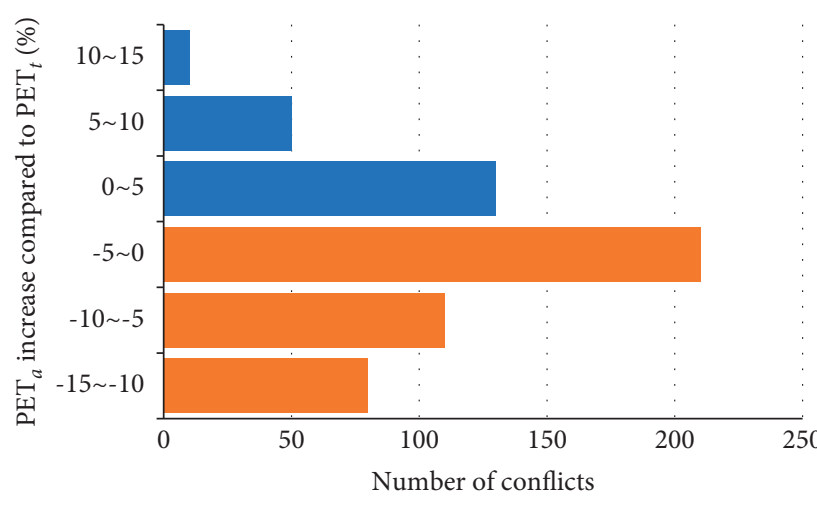

- risk decreasing (32\%)

- risk increasing $(68 \%)$

(b)

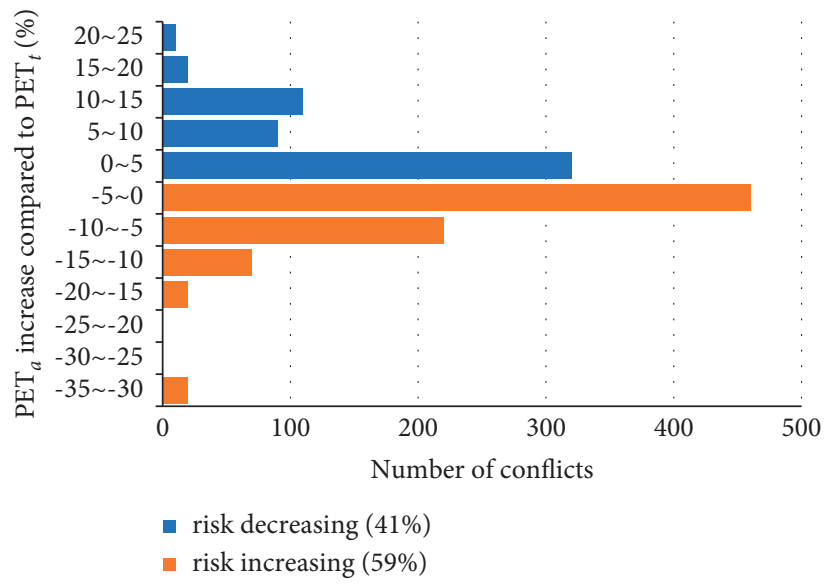

(d)

Figure 8: Continued. 


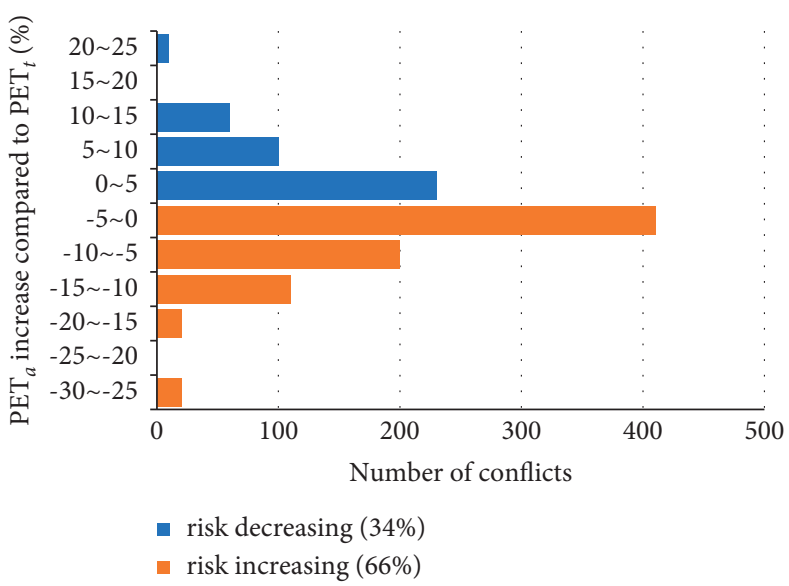

(e)

Figure 8: Conflict risk changes due to left-turn path divergence. (a) $500 \mathrm{veh} / \mathrm{h} / \mathrm{lane}$. (b) $600 \mathrm{veh} / \mathrm{h} / \mathrm{lane}$. (c) $700 \mathrm{veh} / \mathrm{h} / \mathrm{lane}$. (d) $800 \mathrm{veh} / \mathrm{h} /$ lane. (e) $900 \mathrm{veh} / \mathrm{h} / \mathrm{lane}$.

TABle 3: Estimated delay increase due to left-turn path divergence.

\begin{tabular}{|c|c|c|c|c|c|}
\hline Traffic demand (veh/h/lane) & 500 & 600 & 700 & 800 & 900 \\
\hline Total increased delay (sec) & 16.19 & 19.41 & 26.18 & 34.52 & 40.06 \\
\hline Decreased intersection throughput (veh/h) & 31.66 & 45.54 & 71.67 & 108.01 & 141.02 \\
\hline
\end{tabular}

vehicles and adjusting vehicle states to avoid collision. When the actual PET is smaller than the theoretical one assumed by the IM due to the path divergence, CAVs might take riskaverse behaviors such as decelerating due to the increased conflict risk, leading to the drop in travelling speed as well as the intersection efficiency. When the actual PET is larger than the theoretical one, however, CAVs usually follow the schedules while maintaining their safety, and hence, travelling efficiency would not increase. The total delay increase caused by the path divergence is estimated and demonstrated in Table 3, as well as the intersection throughput decline translated from the difference between $\mathrm{PET}_{a}$ and $\mathrm{PET}_{t}$. As observed, the delay increment and the throughput decrement both increase with the increase in traffic demand, indicating that the higher the traffic demand is, the larger effect the path divergence has on the intersection operation efficiency.

\section{Conclusions and Future Directions}

The variations in left-turn paths are usually neglected in previous research, which, however, might affect the safety and efficiency of CIM implementation. We conducted the initial study to analyse the effects by the simulation-based evaluation, including two approaches: the scenario-based approach of different left-turn paths and the PET-based approach with path divergence. Six typical left-turn paths are selected to represent the path variation interval, and the corresponding parameter space of mathematical models is discretized for simulations. In the former approach, the average performance and robustness of schedules obtained with different left-turn paths as nominal paths are evaluated and based on these indicators, using Path E2 as the nominal path is the best choice in terms of the optimality and robustness of solutions. On this basis, further simulation is conducted with Path E2 as the nominal path, and PETs of conflicts are calculated, respectively, with nominal path and actual path to evaluate the effects of path divergence on intersection safety and travelling efficiency. The results show that the number of serious conflicts increases significantly, as well as the overall conflict risk and vehicle delay due to the path divergence. The safety and efficiency deteriorations indicate that even with nominal path of highest robustness, the variations in left-turn paths still need more consideration in the current CIM methods.

The variation in vehicle paths might also affect the safety and efficiency of traffic infrastructure operation where both conflicting movements and turning movements exist, such as the merging roadway scenario. The CIM problem and the merging control problem are very similar in nature, and most of the approaches proposed for CIM can be easily adapted for merging coordination. The similar issue caused by the variation in turning paths of on-ramp vehicles should also be investigated, where the simulation-based evaluation method proposed in this research can be applied.

Work in progress is to further develop the proposed approach by modelling the continuous variation of vehicle paths and paths of irregular graphics to better understand the effects of real vehicle paths on the feasibility of current CIM algorithms. Besides, efforts are also put into combining vehicle collision-avoidance planning of actual turn paths and CIM optimal schedules. 


\section{Data Availability}

The data used to support the findings of this study are available from the corresponding author upon request.

\section{Conflicts of Interest}

The authors declare that they have no conflicts of interest.

\section{Acknowledgments}

This research was supported by the projects of the National Natural Science Foundation of China (nos. 61803083 and 51925801), "the Fundamental Research Funds for the Central Universities," and the Postgraduate Research \& Practice Innovation Program of Jiangsu Province (KYCX17_0140).

\section{References}

[1] K. Dresner and P. Stone, "A multiagent approach to autonomous intersection management," Journal of Artificial Intelligence Research, vol. 31, pp. 591-656, 2008.

[2] J. Lee and B. Park, "Development and evaluation of a cooperative vehicle intersection control algorithm under the connected vehicles environment," IEEE Transactions on Intelligent Transportation Systems, vol. 13, no. 1, pp. 81-90, 2012.

[3] J. Lee, B. Park, K. Malakorn, and J. So, "Sustainability assessments of cooperative vehicle intersection control at an urban corridor," Transportation Research Part C: Emerging Technologies, vol. 32, pp. 193-206, 2013.

[4] Q. Jin, G. Wu, K. Boriboonsomsin, and M. Barth, "Platoonbased multi-agent intersection management for connected vehicle," in Proceedings of the IEEE Conf. Intell. Transp. Syst. Proceedings, ITSC, pp. 1462-1467, IEEE, The Hague, Netherlands, 6 October 2013.

[5] M. W. Levin and D. Rey, "Conflict-point formulation of intersection control for autonomous vehicles," Transportation Research Part C: Emerging Technologies, vol. 85, no. September, pp. 528-547, 2017.

[6] S. A. Fayazi and A. Vahidi, "Mixed-integer linear programming for optimal scheduling of autonomous vehicle intersection crossing," IEEE Transactions on Intelligent Vehicles, vol. 3, no. 3, pp. 287-299, 2018.

[7] C. Yu, W. Sun, H. X. Liu, and X. Yang, "Managing connected and automated vehicles at isolated intersections: from reservation- to optimization-based methods," Transportation Research Part B: Methodological, vol. 122, pp. 416-435, 2019.

[8] Z. Li, Q. Wu, H. Yu et al., "Temporal-spatial dimension extension-based intersection control formulation for connected and autonomous vehicle systems," Transportation Research Part C: Emerging Technologies, vol. 104, no. April, pp. 234-248, 2019.

[9] H. Yu, P. Liu, Y. Fan, and G. Zhang, "Developing a decentralized signal control strategy considering link storage capacity," Transportation Research Part C: Emerging Technologies, vol. 124, Article ID 102971, 2021.

[10] W. Li, Y. Li, H. Deng, and L. Bao, "Planning of electric public transport system under battery swap mode," Sustainability, vol. 10, no. 7, pp. 2528-7, 2018.

[11] A. A. Malikopoulos, C. G. Cassandras, and Y. J. Zhang, "A decentralized energy-optimal control framework for connected automated vehicles at signal-free intersections," Automatica, vol. 93, pp. 244-256, 2018.

[12] Y. Wu, H. Chen, and F. Zhu, "DCL-AIM: decentralized coordination learning of autonomous intersection management for connected and automated vehicles," Transportation Research Part C: Emerging Technologies, vol. 103, no. April, pp. 246-260, 2019.

[13] Y. Zhang and C. G. Cassandras, "Decentralized optimal control of Connected Automated Vehicles at signal-free intersections including comfort-constrained turns and safety guarantees," Automatica, vol. 109, Article ID 108563, 2019.

[14] C. Dong, H. Wang, Y. Li, W. Wang, and Z. Zhang, "Route control strategies for autonomous vehicles exiting to offramps," IEEE Transactions on Intelligent Transportation Systems, vol. 21, no. 7, pp. 3104-3116, 2020.

[15] Y. Zheng, B. Ran, X. Qu, J. Zhang, and Y. Lin, "Cooperative lane changing strategies to improve traffic operation and safety nearby freeway off-ramps in a connected and automated vehicles environment," IEEE Transactions on Intelligent Transportation Systems, vol. 21, no. 11, pp. 4605-4614, 2020.

[16] J. Wu, B. Kulcsár, S. Ahn, and X. Qu, "Emergency vehicle lane pre-clearing: from microscopic cooperation to routing decision making," Transportation Research Part B: Methodological, vol. 141, pp. 223-239, 2020.

[17] J. Wu, S. Ahn, Y. Zhou, P. Liu, and X. Qu, "The cooperative sorting strategy for connected and automated vehicle platoons," Transportation Research Part C: Emerging Technologies, vol. 123, Article ID 102986, 2021.

[18] T. Urbanik et al., Signal Timing Manual, Transportation Research Board, Washington, D.C., 2nd edition, 2015.

[19] Y. Guo, P. Liu, Y. Wu, and J. Chen, "Evaluating how rightturn treatments affect right-turn-on-red conflicts at signalized intersections," Journal of Transportation Safety \& Security, vol. 12, no. 3, pp. 419-440, 2020.

[20] J. Wu et al., "Stationary condition based performance analysis of the contraflow left-turn lane design considering the influence of the upstream intersection," Transportation Research Part C: Emerging Technologies, vol. 123, Article ID 102919, 2021.

[21] K. Fitzpatrick, M. P. Pratt, and R. Avelar, "Speeds of rightturning vehicles at signalized intersections during green or yellow phase," Transportation Research Record: Journal of the Transportation Research Board, vol. 2675, no. 10, pp. 503-515, May 2021.

[22] Z. Ding, C. Sun, M. Zhou, Z. Liu, and C. Wu, "Intersection vehicle turning control for fully autonomous driving scenarios," Sensors, vol. 21, no. 12, pp. 3995-12, 2021.

[23] W. K. M. Alhajyaseen, M. Asano, H. Nakamura, and D. M. Tan, "Stochastic approach for modeling the effects of intersection geometry on turning vehicle paths," Transportation Research Part C: Emerging Technologies, vol. 32, pp. 179-192, 2013.

[24] Z. Pu, Z. Li, R. Ke, X. Hua, and Y. Wang, "Evaluating the nonlinear correlation between vertical curve features and crash frequency on highways using random forests," Journal of Transportation Engineering, Part A: Systems, vol. 146, no. 10, Article ID 04020115, 2020.

[25] Z. Pu, Z. Cui, S. Wang, Q. Li, and Y. Wang, “Time-aware gated recurrent unit networks for forecasting road surface friction using historical data with missing values," IET Intelligent Transport Systems, vol. 14, no. 4, pp. 213-219, 2020.

[26] K. Gnanasaekaran, S. Kanya, B. Suresh, and S. K. Vasudevan, "Automated radius calculation of a turn for navigation and 
safety enhancement in automobiles," Advances in Intelligent Systems and Computing, vol. 384, no. 1, pp. 327-334, 2016.

[27] W. Li, Z. Pu, Y. Li, and X. Ban, "Characterization of ridesplitting based on observed data: a case study of Chengdu, China," Transportation Research Part C: Emerging Technologies, vol. 100, pp. 330-353, 2019.

[28] M. W. Dawande and J. N. Hooker, "Inference-based sensitivity analysis for mixed integer linear programming," $O p$ erations Research, vol. 48, 1998.

[29] Z. Jia and M. G. Ierapetritou, "Short-term scheduling under uncertainty using MILP sensitivity analysis," Industrial \& Engineering Chemistry Research, vol. 43, no. 14, pp. 37823791, 2004.

[30] Z. Ma, J. Xie, X. Qi, Y. Xu, and J. Sun, "Two-dimensional simulation of turning behavior in potential conflict area of mixed-flow intersections," Computer-Aided Civil and Infrastructure Engineering, vol. 32, no. 5, pp. 412-428, 2017.

[31] L. N. Peesapati, M. P. Hunter, and M. O. Rodgers, "Evaluation of postencroachment time as surrogate for opposing left-turn crashes," Transportation Research Record: Journal of the Transportation Research Board, vol. 2386, no. 1, pp. 42-51, Jan. 2013.

[32] L. N. Peesapati, M. P. Hunter, and M. O. Rodgers, "Can post encroachment time substitute intersection characteristics in crash prediction models?" Journal of Safety Research, vol. 66, pp. 205-211, 2018.

[33] L. Zheng and T. Sayed, "From univariate to bivariate extreme value models: approaches to integrate traffic conflict indicators for crash estimation," Transportation Research Part C: Emerging Technologies, vol. 103, no. April, pp. 211-225, 2019.

[34] C. Wang, Y. Xie, H. Huang, and P. Liu, "A review of surrogate safety measures and their applications in connected and automated vehicles safety modeling," Accident Analysis \& Prevention, vol. 157, Article ID 106157, 2021.

[35] S. Malkhamah, M. Tight, and F. Montgomery, "The development of an automatic method of safety monitoring at Pelican crossings," Accident Analysis \& Prevention, vol. 37, no. 5, pp. 938-946, 2005.

[36] D. Gettman and L. Head, "Surrogate safety measures from traffic simulation models final report," vol. 126, 2003 Publ. No FHWA-RD-03-050.

[37] M. C. Ferris, MATLAB and GAMS: Interfacing Optimization and Visualization Software, MINDS@UW, 1999, https:// minds.wisconsin.edu/handle/1793/64396. 